\title{
Generation of Pathologies for Surgical Training Simulators
}

\author{
Raimundo Sierra ${ }^{1}$, Gabor Székely ${ }^{1}$, and Michael Bajka² \\ 1 Computer Vision Group, ETH Zürich, Switzerland, \\ \{rsierra, szekely\}@vision.ee.ethz.ch \\ 2 Clinic of Gynecology, Dept. OB/GYN, University Hospital of Zürich, Switzerland
}

\begin{abstract}
In the past few years virtual reality based systems have been proposed and realized for many medical interventions. These simulators have the potential to provide training on a wide variety of pathologies. So far, realistic generation of anatomical variance and pathologies have not been treated as a specific issue. We report on a cellular automaton, specially developed to generate macroscopic findings fulfilling the requirements for a sophisticated simulation. The specific pathology investigated are leiomyomas protruding to different extents into the uterine cavity. The automaton presented is part of a virtual reality based hysteroscopy simulator which is currently under development.
\end{abstract}

\section{Introduction}

The rapid development of complex minimal invasive surgery creates a strong demand for risk free training environments. Therefore surgical simulators play an increasingly important role in medical training and education. Increasing computational power, as well as current achievements in the field of interactive computer graphics and virtual reality have already led to the rapid development of more or less sophisticated surgical simulators during the past years.

These simulators demonstrate the possible power of virtual reality based training, however they exhibit many unresolved problems to provide the fidelity needed for effective training. One key issue is the generation of anatomical models for the simulation. Todays simulators use single static organ models to build surgical scenes. This anatomical model is usually derived from an exemplary anatomy, such as MRI datasets of a volunteer, from specially acquired high resolution datasets, like the visible human project, or artificially created with CAD 11,19.

To acquire surgical skills, it is highly desirable that the training scene is different from session to session. The configuration of a surgical scene entails both the anatomy of the healthy organ and the incorporation of pathologies. The goal is to generate anatomical models considering the natural variability of the healthy anatomy and seamlessly integrate a wide spectrum of different pathologies according to the specifications from physicians.

Our current research aims at the development of a hysteroscopy simulator. Hysteroscopy is the visualization of the inner surface of the uterus by performing

T. Dohi and R. Kikinis (Eds.): MICCAI 2002, LNCS 2489, pp. 202-210 2002.

(C) Springer-Verlag Berlin Heidelberg 2002 
a distension of the cavum uteri, realized through a single hull for manipulation and visualization. It makes minimal invasive surgery on the uterus possible and allows the physician to perform a specific treatment under organ saving conditions. Hysteroscopy is the second most often performed endoscopic procedure after laparoscopy in gynecology. Because of the lack of alternatives, training is usually performed during actual intervention, in assistance of an experienced gynecologist.

A single organ model is inherently unable to represent an every day situation of the operating site, thus obstructing the training effect of the simulator. The organs of any two patients will never be alike. Statistical anatomical models, such as the ones used for the incorporation of prior anatomical knowledge into the segmentation process [104] offer an appealing way to handle the variability of healthy human anatomy within the organ models used for simulation.

The other requirement for a reasonably realistic surgical simulator is the ability to provide training on a wide variety of pathological cases. The large number of possible pathologies as well as the enormous range of their manifestations makes a similar statistical approach unreasonable if not impossible. We therefore propose to model the pathologies by their genesis.

In this work, the generation of pathologies for surgical simulators is addressed. The focus will be on myomas that are visible from within the uterine cavity, so called submucosal leiomyomas. Their clinical relevance as well as their relatively well-defined appearance makes them the best candidate to gain a better understanding of the artificial generation of pathologies for surgical simulators.

\section{Myoma Formation}

Uterine (leio-)myomas are found in up to $25 \%-40 \%$ of women in their childbearing years, being the most common benign tumors of the uterus in women over 35 years old. They are composed of smooth muscle and a variable amount of fibrous tissue. Blood supply is provided by one or two large vessels [8].

Most often myomas are classified into four types, depending on their position relative to the uterine wall: the intramural myoma, which is confined to the myometrium; the submucosal myoma, which protrudes into the uterine cavity; the subserosal myoma, which projects off the peritoneal surface of the uterus [14; and the intraligamintary myoma which protrudes into the surrounding ligaments. Both the subserosal or the submucosal myoma may be sessile or pedunculated and the latter can become prolapsed through the cervix into the vagina.

All myoma start growing as intramural myomas and are referred to as such as long as they do not vault the endometrium or the serosa. If the growing direction goes towards the uterine cavity and the endometrium is vault, the myoma becomes a submucosal myoma. With continued growth the myoma can protrude through the uterine wall into the uterine cavity. The latter two cases 
are visible and treatable by hysteroscopy and therefore of main interest for the simulator.

For hysteroscopy more detailed categorizations have been proposed [17. Three types of myomas are discerned, which differ in the size of the intramural portion. Pedunculated myomas are classified as type 0. Myomas forming an acute angle with the surrounding uterine wall are predominantly intracavitary (type I, intracavitary portion $>50 \%$ ), whereas the larger portion of type II myomas is an intramural location, if the angle is obtuse. The type of myoma has implications on the hysteroscopy as only type 0 and some type I myomas are regarded as safely resectable in one session, as the resection should never extend further than the inner border of the myometrium [3].

Leiomyomas may be solitary or multiple and over $90 \%$ are found in the uterine corpus. Five percent arise in the cervix and a smaller number are found in the broad ligament. The size of a myoma can vary from a pearl to as large as a melon. A uterus with multiple myoma may even give the impression of a sack filled with different sized potatoes [14.

Despite the amount of research in this area, the exact etiology of myoma is not known. It is assumed that the genesis is initiated by regular muscle cells with increased growth potential and that the growth of myomas is driven by estrogen. They are thus related to the function of the ovaries. Therefore myomas do not appear before puberty and do not emerge after menopause, when already existing myomas even tend to shrink. In general they grow slowly but continuously until the beginning of menopause [6]. The increase of volume by the factor of two usually takes several months or years. Slow growing myomas tend to be squeezed out by the healthy surrounding muscular meshes. Therefore, they seem to migrate over months or years towards the inner surface (endometrium, submucosal) or towards the outer surface (serosa, subserosal). Fast growing myomas, which are potentially malignant, tend to overwhelm this process by stretching out and thinning the healthy surrounding myometrium. They are able to completely deform the organ's appearance.

A myoma has a much stronger tendency to keep its shape than any of the tissues surrounding it, as it is composed of very dense fibrotic tissue. Therefore, the myoma will be able to grow almost independently from its surroundings by keeping a spherical shape. This holds for both the intramural and the submucosal myoma. The surrounding tissue consists of clustered myometrium. There is no actual capsule around the myoma. The tissue of the myoma as well as the surrounding tissue of the myometrium have a layered structure. This often simplifies the resection of the myomas as they can be peeled off the myometrium [14].

The endometrium is a highly reactive tissue covering the whole uterine cavity as well as protruding myomas of any degree. Therefore the endometrium defines the myomas' visual appearance. The myometrium is an active muscular mesh which exhibits slow waves of contractions. This mechanism extrudes any tumor or foreign body affecting the uterine cavity and finally leads to pedunculated myomas. 
Table 1. List of required and neglected features

\begin{tabular}{|l|l|}
\hline \multicolumn{1}{|c|}{ Required } & Neglected \\
\hline realistic shape & exact cellular interaction [157] \\
fully automatic generation & stability of growth [1] \\
randomness & patient specific modeling [18] \\
provide information for: & biomechanical deformation of \\
- texturing & surrounding tissue[12] \\
$\quad$ - blood perfusion & observation of the growing process [9] \\
incorporation in organ model & \\
\hline
\end{tabular}

For a diagnostic description of a myoma the physician will specify: its location within the uterus (fundal, corporal, or cervical), the degree of protrusion into the cavity (type 0, type I, or type II), and the length of the tree main axes [2].

\section{Modeling Requirements}

The generation of pathologies for surgical simulators has to fulfill a number of application specific requirements, which are summarized in Table 1. Tumor growth has previously been modeled with different objectives in mind, but most of the features of these models can be neglected in this application. The main property needed in a surgical simulator is a realistic appearance of the pathology. A surgical training scenario will be configured by a physician and should not need any additional interaction with a simulator expert. Therefore the generation process has to be fully automatic after initialization. The physician has to be able to specify a desired pathology in medical terminology, i.e. the specification of the pathology type and optionally the definition of size and position. An alternative to the definition of the size is the specification of the tumor's age.

The actual generation procedure can be computed off-line and no modifications of the tumor size or position are needed during simulation. That is, the pathology itself does not change during intervention, but of course it might be altered by the trainee, for example by cutting.

In respect of the implementation, the goal is to incorporate a wide range of variations in size, geometry and position within one framework. This demands a nondeterministic model. Once the shape of the pathology has been generated, additional properties that are needed by the simulator have to be added, such as the texturing, the blood perfusion and the biomechanical properties. At the end of the generation process, the tumor has to be seamlessly incorporated into the organ model.

\section{Implementation}

Cellular automata are predestinated for the modeling of pathologies, as they imitate the development just by applying the same set of rules multiple times. 
Once a cellular automaton is able to generate a pathology in one of its most developed stages, any of the intermediate stages can be obtained with no additional effort. The main advantages when using a cellular automaton are the simplicity and extendibility of the implementation. Rules can easily be added, removed or modified. Computational stability is intrinsically a part of a cellular automaton [5]. The synthesis of a cellular automaton is equivalent to the seeking of a minimal set of rules that allows to model a certain behavior. There will always be a trade-off between realism and tractability of the model.

Real myomas consist of millions of cells which is more than can reasonably be modeled in a computer simulation. An exact modeling of single cells implies also the use of a non-regular mesh 9]. This approach may be used to model exactly the behavior of a tumor in its very early stage, but it will not be manageable in the orders of magnitude considered. Thus, the actual value in a node of the cellular automaton can be regarded as a cell conglomeration rather than as a single cell.

The implemented cellular automaton comprises two cell types and the background or cell-free space. The first cell type is the tissue which consists of the muscle cells of the myometrium, the second cell type represents the tumor cells. The background describes the uterine cavity. Additional factors can be added, e.g. to model the influence of the contraction of the uterine muscles. A single node with a tumor component is enough to initiate the growing process of a myoma.

The cellular automaton is defined by a regular, three dimensional, cubic lattice $\mathcal{L}$, an interaction neighborhood template $\mathcal{N}_{b}$, the set of elementary states $\mathcal{E}$, and the local space- and time-independent transition rules $\mathcal{R}_{i}$. The local rules are either probabilistic or deterministic.

As the cell of the automaton does not model a biological cell, the term node is used to avoid misinterpretation. A node is specified by its position $p=(x, y, z)$ in the lattice. The neighborhood template $\mathcal{N}_{b}$ specifies the nodes that influence the state of the node under scrutiny. In the automaton described $\mathcal{N}_{b}$ is rule dependent and can be either a 6 -neighborhood $\left(\mathcal{N}_{6}\right.$, von Neumann neighborhood) or a 26-neighborhood $\left(\mathcal{N}_{26}\right.$, Moore neighborhood). Whenever possible the smaller neighborhood was selected.

The set of elementary states $\mathcal{E}_{\text {tumor }}$ for the tumor is upper bounded by 1 and defined as a multiple of the predefined step $\Delta=\frac{1}{n}, n \in \mathbb{N}$, whereas the set $\mathcal{E}_{\text {tissue }}$ is represented by the floating point values in the range $[0,1]$. With each node, a tumor and a tissue channel can be associated: $c_{\text {tumor }}(p)$ and $c_{\text {tissue }}(p)$, thus tumor and tissue do not exclusively occupy a node. The idea is that only nodes with a value of $c_{\text {tumor }}(p)=1$ are considered to be part of the tumor, while any value smaller than 1 indicates a reactive shell around it. The tissue is initialized with values around 0.5 with decreasing values towards the surface. At least one node needs a tumor component with a value $t_{0} \geq \Delta$ for the growing process to start.

The strict concept of a cellular automaton is relaxed to allow the integration of global knowledge in two aspects: global cost functions can be evaluated and 
a global rule $\mathcal{R}_{\text {global }}$ controlling the application of single rules $\mathcal{R}_{i}$ is introduced. Different rules $\mathcal{R}_{i}$ are applied sequentially in a loop but each rule is applied synchronously on every node. The application of a rule $\mathcal{R}_{i}$ is the transition from time step $t$ to $t+1$ with $\mathcal{R}: \mathcal{E}^{\nu} \rightarrow \mathcal{E}$, where $\nu=\left|\mathcal{N}_{b}\right|$.

A first rule $\mathcal{R}_{\text {grow }}$ determines the growing process of the tumor:

$$
\mathcal{R}_{\text {grow }}: \quad c_{\text {tumor }}(p)_{t+1}=\min \left[1, c_{\text {tumor }}(p)_{t}+\Delta\right]
$$

A node will receive a tumor component with a certain probability if one of its neighbors in $\mathcal{N}_{26}$ has a tumor component. If the neighbor is a direct one, i.e. in $\mathcal{N}_{6}$, the probability of becoming a tumor node is close to one. Otherwise the probability is much smaller. Once a tumor component is in a node it is continuously incremented by the same rule. Three objectives are modeled with this rule; the spherical shape of the myoma, the inhomogeneity of the surface, and the reactive shell around the tumor.

A global cost function for the current tumor position is computed. For each node with a tumor component, the amount of tissue as well as the gradient value for its neighbors in $\mathcal{N}_{6}$ are separately added and summed to six different overall costs $\mathcal{C}_{i}$. The rule $\mathcal{R}_{\text {moving }}$ then moves the tumor into the direction $d$ corresponding to the optimal cost $\min \left(\mathcal{C}_{i}\right)$.

$$
\mathcal{R}_{\text {moving }}: \quad c_{\text {tumor }}(p)_{t+1}=c_{\text {tumor }}(p-d)_{t}
$$

A third rule $\mathcal{R}_{\text {adaption }}$ models the adaption of the surrounding tissue to the new situation. In a first pass the displacement of the tissue introduced by the growing tumor is modeled. In this rule the incremental property of $\mathcal{R}_{\text {grow }}$ is used.

$$
\begin{array}{ll}
\mathcal{R}_{\text {adaption }}: & c_{\text {tissue }}(p)_{t+1}=\min \left[1, c_{\text {tissue }}\left(\underset{q \in \mathcal{N}_{26}}{\arg \max }\left[c_{\text {tumor }}(q)\right]\right)_{t}+c_{\text {tissue }}(p)_{t}\right] \\
& c_{\text {tissue }}\left(\arg \max _{q \in \mathcal{N}_{26}}\left[c_{\text {tumor }}(q)\right]\right)_{t+1}=0
\end{array}
$$

In a second pass the displacement is propagated into the surrounding area by smoothing the tissue state. This could be represented by an additional rule but for simplicity Gauss filters of variable length are used in the actual implementation.

A final rule $\mathcal{R}_{\text {close }}$ closes the covering hull of the myoma so that no node with a tumor component will ever touch the background in $\mathcal{N}_{26}$. This rule is introduced to ensure that the endometrium always covers the tumor, but can be skipped if the relaxation area is large enough, i.e. by applying $\mathcal{R}_{\text {adaption }}$ several times.

The global rule $\mathcal{R}_{\text {global }}$ defines which rule $\mathcal{R}_{i}$ is applied. This rule is time dependent so that the sequence of rules $\mathcal{R}_{i}$ applied changes during evolution. This allows for modeling a faster movement of the tumor while it is small. As the tumor size increases the rule $\mathcal{R}_{\text {moving }}$ is applied less often to model a slower motion. As soon as the tumor is pedunculated this rule can once again be applied more frequently. 


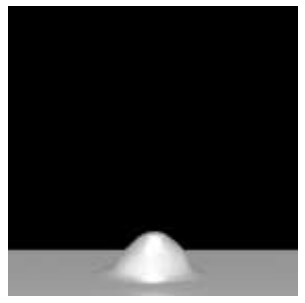

Fig. 1.
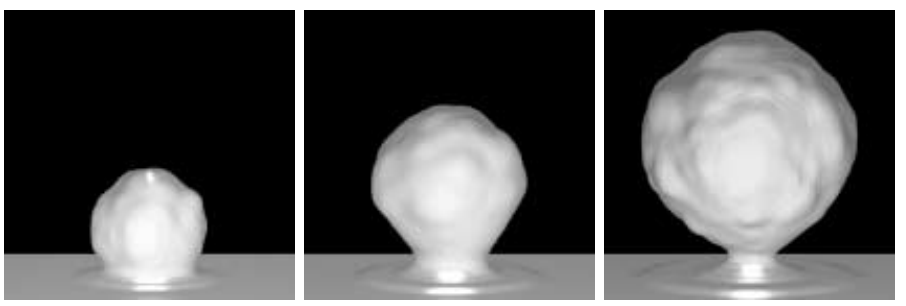

Artificial myoma after 15, 25, 35 and 43 iterations.
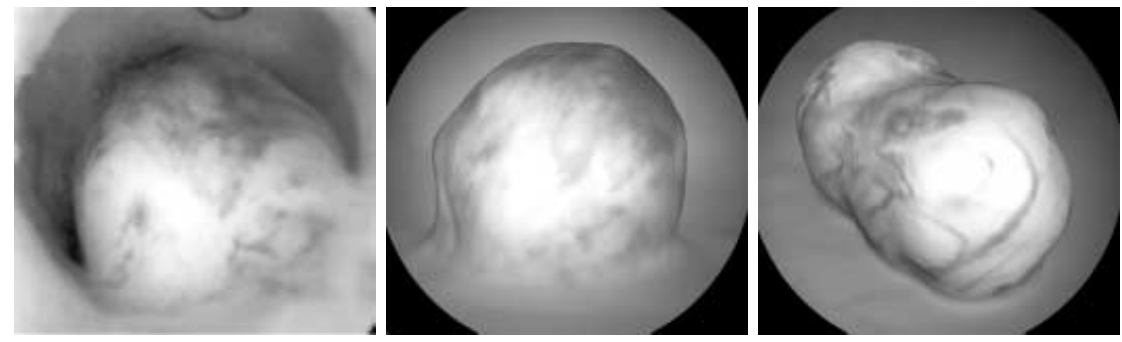

Fig. 2. Comparison of real (left) and two artificial myomas.

As stated in Section 2] the physician will specify the myoma by its location, protrusion and size, so that an explicit time-volume relation cannot be used. Therefore, the Gompertz model for tumor growth, which has been proposed on different occasions $9[15$, is not suitable for this application. Through adaption of the global function $\mathcal{R}_{\text {global }}$ the desired myoma can be generated. By counting the number of applications of rule $\mathcal{R}_{\text {grow }}$ and multiplying this number with the respective probability for $\mathcal{N}_{6}$, one can keep track of the volume. Once the desired value is reached, the procedure exits the main loop.

\section{Results}

This very simple model is sufficient to produce highly satisfactory results and fulfill the requirements of a simulator. Figure 1 shows an exemplary sequence of the growing myoma. The procedure is fully automatic and does not need any interaction with a physician. The volume where the tumor was grown consisted of a cubic lattice with $100^{3}$ nodes. For the conversion to a surface model the marching cubes algorithm was used [13. No additional factors were used and the global rule states that the moving rule is less often applied as the tumor grows.

Figure 2 shows an image of a real myoma on the left which was taken during hysteroscopy. The two following images are synthetic myomas generated with the cellular automaton described. Texture images were taken from real hysteroscopies and mapped on the artificial surfaces. The visual inspection shows a high degree of realism and proves the approach to be suitable for the random 
generation of myomas for hysteroscopy simulation. This and more examples are available for download at http://www.vision.ee.ethz.ch/ rsierra/miccai.

The differentiation between tumor and normal tissue is needed both for the incorporation of the vascularization and the biomechanical properties. The vascularization is confined in the healthy tissue around the myoma. Different biomechanical properties can be assigned to the tumor and the tissue, so that the pathology consists of a stiff inner sphere surrounded by a softer tissue layer.

The structures that can be generated by the cellular automaton proposed are not limited to myomas in the uterine cavity. The automaton can easily be tuned to produce other structures where a growing object is more rigid than the surrounding media.

Validation of the resulting myomas is a major task, as it is in general for any training system. To our knowledge there are currently no other systems that generate artificial pathologies for surgical training devices which could serve as a reference. The structures described have subjectively been analyzed by experienced gynecologist who attested them a very high visual resemblance with actual cases.

In the future validation will be twofold. On one side the training system will be evaluated, which also entails the behavioral and visual aspects of the myoma. Definition of useful metrics for measuring the resemblance as well as the training efficiency are preliminary tasks towards objective measurements. On the other side the growing process of tumors will be more deeply investigated and compared with the cellular automata described.

\section{Conclusion and Future Research}

A cellular automaton that is able to generate submucosal myomas has been described. The generated tumors show a high resemblance to real cases and can be seen as a step forward in the creation of high fidelity simulators.

Future research will on one hand address the generation of other pathologies, and on the other hand incorporate the existing tumor generation model in more complex situations. In the next step, possible pathologies will be extended to incorporate surgically relevant degenerations of the uterus. The incorporation of the existing model in the fundus and corpus of the uterus is straightforward. Close to the fallopian tubes, where the myometrium is much thinner, a myoma will deform more than one surface at a time. In such cases, the incorporation into the organ model has to be further investigated. The generation of a vascular system for the pathology is closely related to the pathology itself. In the future we plan to merge the generation of the pathology with the generation of the vascular system [16].

Acknowledgments This work has been performed within the frame of the Swiss National Center of Competence in Research on Computer Aided and Image Guided Medical Interventions (NCCR CO-ME) supported by the Swiss National Science Foundation. 


\section{References}

1. J. Adam. A simplified mathematical model of tumor growth. Math. Biosci., 81:229-244, 1986.

2. M. Bajka. Empfehlungen zur Gynäkologischen Sonographie. Schweizerische Gesellschaft für Ultraschall in der Medizin, 2001.

3. P. Brandner, K. Neis, and P. Diebold. Hysteroscopic resection of submucous myomas. Contrib Gynecol Obstet., 20:81-90, 2000.

4. Cootes et al. Active shape models - their training and application. Computer Vision and Image Understanding, 61(1):38-59, 1995.

5. S. Dormann. Pattern Formation in Cellular Automaton Models. PhD thesis, Universität Osnabrück, 8.2000.

6. F.H.Netter. Farbatlanten der Medizin, Band 3: Genitalorgane. Georg Thieme Verlag, Stuttgart, New York, second edition, 1987.

7. H. Greenspan. On the growth and stability of cell cultures and solid tumors. $J$. theor. Biol., 56:229-242, 1976.

8. A. Heuck and M. Reiser. Abdominal and Pelvic MRI. Springer, 2000.

9. A. Kansal et al. Simulated brain tumor growth dynamics using a three-dimensional cellular automaton. J. theor. Biol., 203:367-382, 2000.

10. Kelemen et al. Elastic model-based segmentation of 3-D neororadiological data sets. IEEE Transactions on Medical Imaging, 18(10):828-839, 1999.

11. C. Kuhn. Modellbildung und Echtzeitsimulation deformierbarer Objekte zur Entwicklung einer interaktiven Trainingsumgebung für Minimal-Invasive Chirurgie. Forschungszentrum Karlsruhe GmbH, Karlsruhe, 1997.

12. S. Kyriacou et al. Nonlinear elastic registration of brain images with tumor pathology using a biomechanical model. IEEE Transactions on Medical Imaging, 18(7):580-592, 1999.

13. W. Lorensen and H. Cline. Marching cubes: A high resolution 3D surface construction algorithm. Computer Graphics, 21(4):163-170, 7.1987.

14. Pschyrembel, Strauss, and Petri. Praktische Gynäkologie für Studium, Klinik und Praxis. de Gruyter, Berlin, New York, fifth edition, 1990.

15. A. Qi et al. A cellular automaton model of cancerous growth. J. theor. Biol., 161:1-12, 1993.

16. D. Szczerba. Macroscopic modelling of vascular systems. Submitted to MICCAI, 2002.

17. K. Wamsteker, M. Emanuel, and J. de Kruif. Transcervical hysteroscopic resection of submucous fibroids for abnormal uterine bleeding: Results regarding the degree of intramural extension. Obstet Gynecol, 82:736-740, 1993.

18. R. Wasserman and R. Acharya. A patient-specific in vivo tumor model. Math. Biosci., 136:110-140, 1996.

19. LASSO Project. http://www.vision.ee.ethz.ch/projects/Lasso/start.html, 2001. 